\title{
Influence of Silver Incorporation on the Structural and Electrical Properties of Diamond-Like Carbon Thin Films
}

Neeraj Dwivedia,b,1, Sushil Kumar ${ }^{a, *}$, J. David Carey ${ }^{\mathrm{c}}$, R. K. Tripathi ${ }^{\mathrm{a}}$, Hitendra K. Malik ${ }^{\mathrm{b}}$ and M. K. Dalai ${ }^{\mathrm{a}}$

a National Physical Laboratory (CSIR), K.S. Krishnan Road, New Delhi-110 012, India,

${ }^{b}$ Department of Physics, Indian Institute of Technology Delhi, New Delhi-110016, India,

${ }^{c}$ Advanced Technology Institute, University of Surrey, Guildford, GU2 7XH, Surrey, United Kingdom

\begin{abstract}
A simple approach is proposed for obtaining low threshold field electron emission from large area diamond-like carbon (DLC) thin films by sandwiching either Ag dots or a thin Ag layer between DLC and nitrogen containing DLC films. The introduction of silver and nitrogen is found to reduce the threshold field for emission to under $6 \mathrm{~V} / \mu \mathrm{m}$ representing a near $46 \%$ reduction when compared with unmodified films. The reduction in the threshold field is correlated with the morphology, microstructure, interface and bonding environment of the films. We find modifications to the structure of the DLC films through promotion of metal-induced $\mathrm{sp}^{2}$ bonding and the introduction of surface asperities, which significantly reduce the value of the threshold field. This can lead to the next generation large area simple and inexpensive field emission devices.
\end{abstract}

Keywords: diamond-like carbon, silver, field emission, threshold field, Raman spectroscopy, microscopy

* Corresponding Author: E-mail address: skumar@ nplindia.org (Sushil Kumar)

neerajdwivedi6@gmail.com 


\section{INTRODUCTION}

Recently, low dimensional carbon materials, such as carbon nanotubes (CNTs) and graphene, have shown excellent electronic properties and have opened up the possibility to develop new electronics beyond silicon. CNTs and graphene are both highly conducting materials and have been used for the fabrication of electronic devices, though their large scale production is still awaited. CNTs, CNT-composites and graphene all exhibit excellent field emission (FE) characteristics leading to their potential application as X-ray sources for security applications or mobile medical diagnostics or in field emission displays or lighting (FEDs), ${ }^{1-3}$ however, their manufacture for large area electronic applications remains complex. By contrast, other low dimensional carbon materials, such as diamond-like carbon (DLC) thin films can be readily produced over large areas and on substrates held at low (room) temperature. These films possess both $\mathrm{sp}^{3}$ and $\mathrm{sp}^{2}$ bonded carbon and exhibit tunable mechanical, electrical and optical properties. ${ }^{4-15}$ Here, we focus on the FE characteristics of DLC films and suggest ways to reduce the electric field required to initiate emission by adjusting the $\mathrm{sp}^{2}$ carbon phase and surface morphology.

Cold electron field emission is the process in which electrons from a cathode are extracted from a surface through quantum tunneling through the surface potential barrier by the application of an electric field. The threshold field $\left(\mathrm{E}_{\mathrm{T}}\right)$ for emission, an essential material parameter, reflects the onset of emission and for good field emission devices $\mathrm{E}_{\mathrm{T}}$ should to be as low as possible. Previous studies of DLC based field emission devices have shown $\mathrm{E}_{\mathrm{T}}$ can be as high as $10-20 \mathrm{~V} / \mu \mathrm{m}$. For example, llie et al. ${ }^{15}$ have found $\mathrm{E}_{\mathrm{T}}$ in the range $21 \mathrm{~V} / \mu \mathrm{m}$ to $10 \mathrm{~V} / \mu \mathrm{m}$ in amorphous carbon films, whereas Silva et al. ${ }^{16}$ have found $\mathrm{E}_{\mathrm{T}}$ close to $5 \mathrm{~V} / \mu \mathrm{m}$ in nanostructured DLC films having $\mathrm{N}$ contents as high as 11 at.\% and 15 at.\%. Forrest et al. ${ }^{17}$ 
examined the effect of the thickness of hydrogenated DLC (a-C:H) and nitrogenated tetrahedral amorphous carbon (ta-C:N) films on the field emission characteristics of their films and found high values of $E_{T}$ in a-C:H films which varied between $60 \mathrm{~V} / \mu \mathrm{m}$ and $10 \mathrm{~V} / \mu \mathrm{m}$, though lower values were found in ta-C:N films. Carey et al. ${ }^{18-21}$ explored the effect of self-bias and nitrogen content on electronic properties of a-C:H films and the effect on the value of $\mathrm{E}_{\mathrm{T}}$ and found variation of $E_{\mathrm{T}}$ from about $26 \mathrm{~V} / \mu \mathrm{m}$ to $10 \mathrm{~V} / \mu \mathrm{m}$ by changing the negative self-bias. They suggested the importance of the size and concentration of $\mathrm{sp}^{2}$ clusters on field emission from DLC films and have proposed a description that explains the origin of the low threshold field based on internal field enhancement and dielectric inhomogeneity of conductive $\mathrm{sp}^{2}$ clusters embedded in an insulating $\mathrm{sp}^{3}$ matrix. They further discussed the effect of current annealing and hysteresis on field emission from DLC films by changing of the insulating $\mathrm{sp}^{3}$ phase into conductive $\mathrm{sp}^{2}$ carbon. ${ }^{22}$

Despite extensive studies of the FE properties of DLC films, values of $\mathrm{E}_{\mathrm{T}}$ remain high especially from films deposited by plasma enhanced chemical vapor deposition (PECVD). As a consequence there has been considerable interest in developing ways to reduce the value of $\mathrm{E}_{\mathrm{T}}$ by introducing nanostructured surfaces to increase the field enhancement and by doping to change the relative $\mathrm{sp}^{2} / \mathrm{sp}^{3}$ ratio. Nitrogen inclusion in DLC films is usually accompanied by changes in the $\mathrm{sp}^{2}$ phase $^{16}$ and improvements in transport. For example, Khan et al. ${ }^{23}$ used low mass ion implantation to convert $\mathrm{sp}^{3}$ to $\mathrm{sp}^{2}$ carbon and reported an improvement in conduction as $\mathrm{sp}^{3}$ carbon bonding was changed to $\mathrm{sp}^{2}$ carbon. Ahmed et $a .^{24}$ observed a reduction in the value of threshold field for emission upon silver incorporation in the DLC matrix; metal inclusion can help to promote $\mathrm{sp}^{2}$ bonding. Other metals such as $\mathrm{Li}, \mathrm{Cs}, \mathrm{Ca}, \mathrm{Sr}$ and $\mathrm{Ba}$ have also been incorporated in the DLC matrix to lower $\mathrm{E}_{\mathrm{T}}$ value $\mathrm{e}^{25}$, however, metal incorporation requires a 
hybrid system involving both sputtering and PECVD units which may result in poisoning of the targets. ${ }^{26}$ By contrast, the use of a metal interlayer prior to carbon film growth can be a simple and effective way to reduce the value of $\mathrm{E}_{\mathrm{T}}$ of DLC thin films and may also help to change the carbon overlayer from their amorphous to nanostructured phases. ${ }^{27}$ Lately, we have reduced the value of $\mathrm{E}_{\mathrm{T}}$ of DLC films from $14.8 \mathrm{~V} / \mu \mathrm{m}$ to $11.4 \mathrm{~V} / \mu \mathrm{m}$ by employing a $\mathrm{Cu}$ interlayer ${ }^{28}$, through the use of a hybrid sputtering and PECVD system. As a result, we have chosen to study the FE characteristics of DLC films in the presence of silver (both dots and a thin interlayer). Silver was chosen due to its low melting point, high conductivity and high stability characteristics. We will also explore the effect of nitrogen addition to demonstrate how changes in the $\mathrm{sp}^{2}$ phase depend on the impurity atom and presence of a metal. We correlate the observed changes in the field emission characteristics with the changes in morphology, microstructure, bonding environment and also with the interface properties of these films.

\section{MATERIALS AND METHODS}

2.1 Deposition of DLC and modified DLC thin films. Pure and nitrogen incorporated DLC thin films were deposited at a base pressure of $10^{-5}$ Torr on well cleaned $n$ - and $p$-type silicon substrates using a radio frequency (13.56 MHz) PECVD system. The working pressure of acetylene $\left(\mathrm{C}_{2} \mathrm{H}_{2}\right)$, argon $(\mathrm{Ar})$ and nitrogen $\left(\mathrm{N}_{2}\right)$ was kept at $2.4 \times 10^{-3}$ Torr and a fixed self-bias of $-135 \mathrm{~V}$ is used for the deposition of all the films. A pure DLC film (sample ND-1) was grown at a working pressure of $2.4 \times 10^{-3}$ Torr, obtained by feeding $\mathrm{C}_{2} \mathrm{H}_{2}$ gas to change the pressure to 7.5 $\times 10^{-4}$ Torr and then Ar gas to change the pressure to $2.4 \times 10^{-3}$ Torr. A nitrogen containing DLC film (DLC:N, sample ND-2) was deposited at a working pressure of $2.4 \times 10^{-3}$ Torr obtained by first feeding $\mathrm{C}_{2} \mathrm{H}_{2}$ gas and then $\mathrm{N}_{2}$ gas. Similar DLC and DLC:N films were also produced on Ag dots as shown in Fig. 1. First, bare Si substrates (Figure 1a) are taken and then Ag dots 
(Figure 1b) are deposited over them with the help of a mask using thermal evaporation. The diameter of each $\mathrm{Ag}$ dot is found to be around $500 \mu \mathrm{m}$ and the separation between the edges of the dots is $\sim 500 \mu \mathrm{m}$. Subsequent to Ag deposition, DLC and DLC:N layers are grown on the Ag dots (Figure 1c). Sample ND-3 is obtained when a DLC layer is deposited over the Ag dots and sample ND-4 is obtained when a DLC:N film is deposited over the Ag dots. The samples show the formation of peaks and valleys over the surface (Figure 1d). Owing to a sufficient separation between the Ag dots, two different regions are formed when the DLC or DLC:N layers are grown. One region is located between the Ag dots and possesses a configuration of Si/DLC or $\mathrm{Si} / \mathrm{DLC}: \mathrm{N}$ (called the valley region) and the other region corresponds to a configuration of $\mathrm{Si} / \mathrm{Ag}$ dots/DLC or Si/Ag dots/DLC:N (called the peak region). Besides using Ag dots, Ag/DLC and $\mathrm{Ag} / \mathrm{DLC}: \mathrm{N}$ bilayers are also deposited for which the $\mathrm{Ag}$ interlayer is deposited on the $\mathrm{Si}$ substrates using thermal evaporation followed by the deposition of DLC and DLC:N layers over them. When the DLC (DLC:N) film deposited on a continuous Ag layer, sample ND-5 (sample ND-6) is obtained.

2.2 Characterization methods employed. All the films are characterized for their morphological and structural properties by scanning electron microscopy (SEM) (JEOL, JSM35), atomic force microscopy (AFM) (Veeco V) and micro Raman spectroscopy (Renishaw inVia Reflex micro Raman spectrometer attached to an air cooled argon ion laser). Compositional analysis was carried out using time-of-flight secondary ion mass spectroscopy (TOF-SIMS) (TOF-SIMS 5 of ION-TOF GmBH, Germany) and energy dispersive X-ray analysis (EDAX). The field emission characteristics were examined using a Keithley software controlled field emission measurement unit (Keithley 2410). The thickness of the films was determined using a Taylor-Hobson Talystep and found to be about 160 and $140 \mathrm{~nm}$ in DLC (ND-1) and DLC:N 
(ND-2) films, respectively. The thickness of either Ag dots or Ag layer is found to be about 55 nm. For micro Raman measurements, $514 \mathrm{~nm}$ was used for excitation source. ToF-SIMS measurements were performed under ultra-high vacuum conditions with a pressure of $3.75 \times 10^{-}$

10 Torr. Pulsed primary ions from a $25 \mathrm{keV}$ Bi liquid-metal ion gun (LMIG) were used to bombard the sample surface to create secondary ions. The primary ion dose density was approximately $10^{14}$ ions $\mathrm{cm}^{-2}$ for all investigated samples. The sputtering was carried out by using a $500 \mathrm{eV} \mathrm{O}_{2}$ source to remove the first few layers from the sample surface. The sputtering and the analysis area were kept at $200 \times 200 \mu \mathrm{m}^{2}$ and $70 \times 70 \mu \mathrm{m}^{2}$, respectively for all the samples investigated. The overall depth resolution is better than $1 \mathrm{~nm}$. The field emission measurements were carried out at a base pressure of $10^{-7}$ Torr using a Keithley high voltage source. The sample was placed at the cathode and an ITO coated glass slide was used as the anode. Only samples deposited on $n$-type Si were used for field emission characterization.

\section{RESULTS AND DISCUSSION}

Figures $2 \mathrm{a}$ and $2 \mathrm{~b}$ show the SEM images of the DLC and DLC:N films (samples ND-1 and ND2), and show the formation of a smooth, texture less surface; a characteristic of amorphous carbon thin films. However, when DLC and DLC:N films are grown over the Ag dots on Si, nano- and micro-structures are realized in the resultant Ag dot/DLC (sample ND-3) and Ag dot/DLC:N (sample ND-4) films. Figure 2c shows a larger area SEM image of a DLC film deposited on Ag dots (sample ND-3) and Figure 2d shows a micrograph of sample ND-3 captured from the valley region located between the Ag dots with the corners of the dots visible. This central region is found to be amorphous in nature which is consistent with the structure of sample ND-1. However, the region possessing a $\mathrm{Si} / \mathrm{Ag}$ dot/DLC configuration (peak region) 
reveals a change in the morphology and the formation of small carbon nanostructures are found (Figure 2e). When a DLC:N film is grown over the Ag dots (Ag dot/DLC:N film, sample ND-4), the same two types of regions are found. The valley region (Figure $2 \mathrm{f}$ ) is found to be smooth in nature, however, the peak region shows the formation of a different micro-structured morphology (Figure 2g). In contrast to the peak region of sample ND-3, here the formation of a chain-like structure is observed. The analysis of samples ND-3 and ND-4 infers that the Ag dots help to change the amorphous structure of the carbon film, observed in samples ND-1 and ND-2 into micro- and nanostructures of carbon. The SEM analysis of DLC:N film grown over the thin Ag layer (configuration Si/Ag layer/DLC:N, sample ND-6) was also conducted and shown in Figure 2h. This sample reveals the formation of well organized, uniform and highly dense spherical carbon nanostructures. The average size of carbon nanoparticles in sample ND-6 is found to be between $70-80 \mathrm{~nm}$. As metal and nitrogen both help increasing the graphite-like $\mathrm{sp}^{2}$ bonding, it is expected that such micro and nano-structures have a higher $\mathrm{sp}^{2}$ carbon content, which is confirmed by micro-Raman analysis (discussed later).

Atomic force microscopy (AFM) analysis has been performed to investigate the morphology and roughness of the DLC and modified DLC films. Figure 3a shows an AFM 3D micrograph of sample ND-1, which clearly reveals a mirror smooth surface, in agreement with the SEM micrograph of the sample ND-1. AFM measurements of sample ND-3 are taken from the peak region (Fig. 3b) and valley region (Fig. 3c). Here, the DLC layer grown over the Ag dot (peak region) clearly reveals the formation of nanostructures, whereas the area containing the configuration of $\mathrm{Si} / \mathrm{DLC}$ (valley region) located between the Ag dots is found to be mirror smooth. Figure 3d is the AFM 3D micrograph of the sample ND-3 taken after the FE measurement and reveal structural changes (discussed later) of the surface. The AFM micrograph 
of the sample ND-6 has also been captured, Fig. 3e, and reveals a uniform and high density distribution of carbon nanoparticles on the surface. Hence, it is concluded that both $\mathrm{Ag}$ dots and the Ag layer help in the formation of a carbon nanostructured surface. AFM has also been used for surface roughness analysis. The root-mean-square roughness $\left(\mathrm{R}_{\mathrm{q}}\right)$ of sample ND-1 is found to be $0.12 \mathrm{~nm}$ justifying its mirror smooth description. In the case of the sample ND-3, we have measured $\mathrm{R}_{\mathrm{q}}$ from three different regions; in the valley region a value of $0.16 \mathrm{~nm}$ is found which increases to $1.5 \mathrm{~nm}$ in the peak region owing to the introduction of metallic $\mathrm{Ag}$ dots below the DLC layer. The value of $\mathrm{R}_{\mathrm{q}}$ in the conditioned area, post field emission testing, is found to be larger at $64 \mathrm{~nm}$. Finally, in sample ND-6 having DLC:N film on the Ag layer the roughness is found to be $4.8 \mathrm{~nm}$. Since metal films are rougher than the DLC and DLC:N films, the roughness analysis implies that the introduction of $\mathrm{Ag}$ (dots or layers) increases the overall roughness of the surface of the film. The grain size of the sample ND-6 is also estimated by AFM and it is found to be about $80 \mathrm{~nm}$, which agrees well with the SEM analysis.

TOF-SIMS measurements were carried out to investigate the constituents of the films as well as to study the diffusion of atoms into the Si substrate. The depth profiles for samples ND-1 and ND-2 are presented in Figs. 4a and 4b, respectively. These figures clearly reveal the diffusion of $\mathrm{C}, \mathrm{H}$ and $\mathrm{N}$ atoms into the Si substrate. However, among the two samples, the diffusion of C and $\mathrm{H}$ atoms into $\mathrm{Si}$ is found to be higher in sample $\mathrm{ND}-1$. Among $\mathrm{H}$ and $\mathrm{C}$ atoms, $\mathrm{H}$ diffuses deeper owing to its lower atomic mass. Examination of the depth profile of the other samples suggests that the introduction of nitrogen has reduced the diffusion of $\mathrm{C}$ and $\mathrm{H}$ into Si. A consequence of this may be to reduce the interfacial mismatch and interfacial stress and help to improve the adhesion of film to the substrate. Depth profiles of silver modified DLC and DLC:N films are also recorded for samples ND-3, ND-5 and ND-6. The depth profile of sample ND-3 is 
recorded both over and away from the silver region. The depth profile of the valley region, where the DLC layer is only over the substrate Si (no Ag dot region), is presented in Fig. 4c. The profile of the valley region is similar to the depth profile of sample ND-1 (Fig. 4a) and exhibits significant diffusion of $\mathrm{C}$ and $\mathrm{H}$ atoms into the substrate. The depth profile of the peak region is presented in Fig. 4d and reveals that all the constituents $\mathrm{Ag}, \mathrm{C}$ and $\mathrm{H}$ of this region diffuse into the substrate. The depth profiles for samples ND-5 and ND-6 are presented in Figs. 4e $-4 f$. Here, it can be seen that the diffusion of $\mathrm{C}$ and $\mathrm{H}$ in sample ND-5 is found to be comparable to that of sample ND-1. The diffusion of $\mathrm{C}$ and $\mathrm{H}$ is further reduced in sample ND-6 due to nitrogen introduction. It can be seen that comparing samples ND-5 and ND-6 the diffusion of Ag into Si is lower in sample ND-6, which may be due to the presence of nitrogen in this sample. The analysis of the TOF-SIMS results reveals the presence of all constituents of the samples and a reduction of $\mathrm{C}$ atom diffusion into the substrate due to nitrogen, $\mathrm{Ag}$ dots or $\mathrm{Ag}$ interlayer addition. Finally, the approximate percentage of nitrogen in sample ND-2 is calculated by taking the average intensity ratios. The nitrogen content in a sample ND-2 is found to be about 5 at.\%. The estimated nitrogen content by ToF-SIMS agrees well with the nitrogen content as estimated by EDAX which is estimated to be about 4.7 at.\%.

Raman spectroscopy is found to be one of the most important non-destructive characterization tools for low dimensional carbon nanomaterials and is able to probe the $\mathrm{sp}^{2}$ and $\mathrm{sp}^{3}$ carbon phases separately. The DLC and modified DLC films contain diamond-like $\mathrm{sp}^{3}$ and graphite-like $\mathrm{sp}^{2}$ bonding. The room temperature electronic band gap of $100 \%$ crystalline $\mathrm{sp}^{3}$ bonded diamond is around $5.5 \mathrm{eV}$, whereas $100 \% \mathrm{sp}^{2}$ bonded graphite has a zero band gap as the $\pi-\pi^{*}$ bands overlap. In the case of amorphous carbon films visible Raman spectroscopy effectively probes the $\mathrm{sp}^{2}$ phase. Tamor and Vassell ${ }^{29}$, Weiler et al. ${ }^{30}$, Ferrari and Robertson ${ }^{31}$, 
Ferrari et $a l .{ }^{32}$ and Rodil et $a l .{ }^{33}$ have all widely employed micro Raman technique for probing the microstructure of amorphous carbon thin films. Usually visible Raman spectra of DLC films exhibits two important bands, namely the $\mathrm{D}$ (disorder) band near $1350 \mathrm{~cm}^{-1}$ and the $\mathrm{G}$ band near $1550 \mathrm{~cm}^{-1}$. The $\mathrm{D}$ band is associated with the $\mathrm{A}_{1 \mathrm{~g}}$ breathing mode of phonons near the zone boundary and appears in the presence of disorder; this mode is absent in perfect or crystalline graphite. The $\mathrm{G}$ band is the $\mathrm{E}_{2 \mathrm{~g}}$ stretching mode and occurs because of in-plane displacement of $\mathrm{sp}^{2}$ carbon atoms against in-plane restoring forces. ${ }^{31-33}$ The Raman spectra of various DLC and modified DLC films, in the range $1000-2000 \mathrm{~cm}^{-1}$, are depicted in Figure 5a - 5e. The Raman spectrum of each sample reveals the presence of both the D and G peaks. The introduction of nitrogen and $\mathrm{Ag}$ (either dots or layer) results in a shifting of the $\mathrm{D}$ and $\mathrm{G}$ peak positions and a change in the $I_{D} / I_{G}$ ratio. In order to examine the exact $D$ and $G$ peaks positions and their $I_{D} / I_{G}$ ratios, the Raman spectra were fitted with two Gaussian functions. The variation of the evaluated G peak position and $\mathrm{I}_{\mathrm{D}} / \mathrm{I}_{\mathrm{G}}$ ratio for different samples is shown in Figs. $6 \mathrm{a}$ and $6 \mathrm{~b}$, respectively. The $G$ peak position and $\mathrm{I}_{\mathrm{D}} / \mathrm{I}_{\mathrm{G}}$ ratio of sample ND-1 are found to be $1549 \mathrm{~cm}^{-1}$ and 0.41 , respectively, which increases to $1560 \mathrm{~cm}^{-1}$ and 0.69 (sample ND-2); to $1569 \mathrm{~cm}^{-1}$ and 0.85 (sample ND-4); to $1568 \mathrm{~cm}^{-1}$ and 0.9 (sample ND-5) and to $1569 \mathrm{~cm}^{-1}$ and 1.0 , respectively, (sample ND-6). The increased position of the $\mathrm{G}$ peak and the $\mathrm{I}_{\mathrm{D}} / \mathrm{I}_{\mathrm{G}}$ ratio in nitrogen, $\mathrm{Ag}$ dot and Ag layer modified DLC films are attributed to an increase in $\mathrm{sp}^{2}$ bonding.

The emission current-applied field (I-E) curves of the films are shown in Fig. 7a. Before recording the final FE curves, electrical conditioning was performed until the emission current becomes stable. The value of the threshold field $\left(\mathrm{E}_{\mathrm{T}}\right)$ is defined at a current of $\sim 3 \times 10^{-7} \mathrm{~A}$. The DLC film (sample ND-1) shows a $\mathrm{E}_{\mathrm{T}}$ of around $11 \mathrm{~V} / \mu \mathrm{m}$ and about $10.8 \mathrm{~V} / \mu \mathrm{m}$ in DLC:N film (sample ND-2). In the DLC/Ag dot film (sample ND-3), $\mathrm{E}_{\mathrm{T}}$ is $12.7 \mathrm{~V} / \mu \mathrm{m}$ but is significantly 
reduced to $5.9 \mathrm{~V} / \mu \mathrm{m}$ in DLC:N/Ag dot film (sample ND-4). The value of $\mathrm{E}_{\mathrm{T}}$ from the DLC/Ag film (sample ND-5) and DLC:N/Ag film (sample ND-6) are found to be $10.4 \mathrm{~V} / \mu \mathrm{m}$ and 10.1 $\mathrm{V} / \mu \mathrm{m}$, respectively. In order to avoid the possibility of field emission from the Ag dots and Ag layer themselves, FE measurements on samples with either Ag dots or Ag layer deposited on a $n$ Si substrate (without DLC and DLC:N films deposition over them) were also conducted. Up to a maximum field of $20 \mathrm{~V} / \mu \mathrm{m}$ no emission was recorded, which confirms that the emission of the electrons occurs through carbon based overlayer structures with the Ag dots and Ag layers help in the formation of the micro- and nanostructure. The electron emission characteristics of the samples can be fitted (Figure 7b) to the simplified Fowler-Nordheim (F-N) equation,

$$
J=\frac{a(\beta E)^{2}}{\phi} \exp \left(\frac{-b \phi^{3 / 2}}{\beta E}\right)
$$

where $\mathrm{J}$ is the current density, $\Phi$ is taken as the barrier height to emission, $\mathrm{E}$ is the applied electric field, $\beta$ is the field enhancement factor and $a$ and $b$ are constants with the values of 1.56 $\mathrm{x} 10^{-6} \mathrm{~A} \mathrm{eV} \mathrm{V}^{-2}$ and $6.83 \times 10^{7} \mathrm{~V} \mathrm{eV}^{-3 / 2} \mathrm{~cm}^{-1}$, respectively. A good fit to the $\mathrm{F}-\mathrm{N}$ emission mechanism suggests emission of electrons is via tunneling through an approximately triangular front surface potential barrier.

The threshold fields for samples ND-1, ND-2, ND-5 and ND-6 are all similar (10 - 11 V/ $\mu \mathrm{m})$ whereas sample ND-4 has a much lower threshold field $(5.9 \mathrm{~V} / \mu \mathrm{m})$. Samples ND-1 and ND-2 both possess mirror smooth surfaces though the latter has about 5 at. $\%$ N present. Sample ND-3 has some surface roughness in the region with the Ag dots but has a similar flat finish elsewhere. Sample ND-4 has both Ag dots and a textured surface finish and sample ND-6 has a high concentration of closely spaced spherical surface asperities. In relation to the emission from sample ND-6 it is highly likely that the field lines from the anode terminate at the tips of the 
asperities and it is from here that the emission originates. For the other samples it is worth recalling that field emission from DLC films depends on the electronic properties of the films with two generic types of emission mechanism possible. In general samples grown at low selfbias possess a low defect (electron spin resonance) density ${ }^{19}$ as well as low conductivity with a high $\mathrm{H}$ content (and high $\mathrm{C}-\mathrm{H} \mathrm{sp}^{3}$ content) and are sometimes referred to as polymer-like amorphous carbon (PAC) films. ${ }^{20}$ In such films the electron emission is controlled by the properties of the film/substrate contact. Films grown at higher self-biases, such as those in this study, have a higher $\mathrm{sp}^{2}$ content and a higher conductivity ${ }^{21}$ with $\mathrm{sp}^{2}$ clusters embedded in the insulating $\mathrm{sp}^{3}$ matrix. The field lines from the anode terminate on the more conductive elements $\left(\mathrm{sp}^{2}\right.$ carbon) within the film and give rise to a dielectric inhomogeneity ${ }^{20}$, which helps to increase the field enhancement factor and reduce $\mathrm{E}_{\mathrm{T}}$. As a consequence the electron emission properties of mirror smooth films are determined by the concentration and size of the $\mathrm{sp}^{2}$ clusters in the film and the properties of the front surface potential barrier. In the DLC films grown here, a so-called front surface controlled emission of electrons is the most probable emission mechanism with the replacement of emitted electrons not limited by transport through the film. The addition of the $\mathrm{N}$ helps to increase the size of the clusters (as evidenced by the small change in the G peak position) of sample ND-2 compared to sample ND-1 but without changing significantly the $\mathrm{sp}^{2}$ concentration or surface morphology; hence the threshold fields are similar. A higher concentration of $\mathrm{N}$ atoms would be needed to affect the surface morphology.

Samples ND-4, 5 and 6 have a similar Raman G peak position though sample ND-4 has the lowest threshold field. In this case the electron emission is determined by the surface properties (asperities or texture) of the sample. From Fig. 2g and Fig. 2h samples ND-4 and ND6 exhibit a high degree of texturing though the key difference which explains the higher 
threshold field in sample ND-6 is the closeness of the emitting sites. This closeness of the emitters gives rise to a proximity electrostatic screening ${ }^{34}$ of the emitters and reduces the electric field experienced at the tip of the emitters. As a result a higher applied field is required even though the Raman spectra suggest similar electronic properties of the film. Finally, we note that the value of $\mathrm{E}_{\mathrm{T}}$ for sample ND-3 is the highest at $12.7 \mathrm{~V} / \mu \mathrm{m}$, over twice that of sample ND-4. We repeated the field emission measurements of (new) samples of ND-3 and ND-4 and found value of $\mathrm{E}_{\mathrm{T}}$ to be $12.6 \mathrm{~V} / \mu \mathrm{m}$ and $5.8 \mathrm{~V} / \mu \mathrm{m}$, respectively, similar to that found previously. The AFM analysis of sample ND-3, post emission, showed some damage with an rms roughness of $63 \mathrm{~nm}$ suggesting some form of dielectric breakdown was present in this sample. Our study shows that the choice of a low incorporation of $\mathrm{N}$ atoms ( $<5$ at.\%) coupled with the different structures of $\mathrm{Ag}$ (dots or layers) can result in very different electronic and structural properties which in turn control the field induced tunneling of electrons. In this study we have been able to distinguish between electron emissions controlled simply by the internal $\mathrm{sp}^{2}$ phase of the films from emission controlled by surface texturing. The addition of a small amount of $\mathrm{N}$ was insufficient alone to lower the threshold field and neither was the presence of Ag alone enough to adjust the surface morphology; it was only the combination of both that reduced the threshold field to less than $6 \mathrm{~V} / \mu \mathrm{m}$.

\section{CONCLUSIONS}

A variety of DLC and modified DLC films were deposited using the rf-PECVD technique and studied for their structural, compositional, interface and field emission properties. The addition of $\mathrm{Ag}$ dots and $\mathrm{Ag}$ interlayers in the DLC and DLC:N films changes their bulk amorphous to micro and nano-structured morphologies and enhances the $\mathrm{sp}^{2}$ bonding and reduces the diffusion of $\mathrm{C}$ into Si. This suggests that low threshold field emission can be achieved from DLC films by 
the inclusion of $\mathrm{Ag}$ and $\mathrm{N}$ sufficient to change the $\mathrm{sp}^{2}$ phase in the films. Among various samples, the lowest threshold field $(5.9 \mathrm{~V} / \mu \mathrm{m})$ during the electron emission is encountered in the sample ND-4 owing to the presence of a low concentration of nitrogen and with Ag dots which changed the morphology to a textured surface. Finally, owing to excellent field emission properties, these modified DLC films can be potential candidates for next generation, inexpensive and large area field emission devices.

\section{ACKNOWLEDGEMENTS}

Authors are grateful to the Director, National Physical Laboratory, New Delhi (India) for his kind support. Authors wish to thank Mr. Ishpal, Ms. Kalpana Lodhi, Mr. Sandeep, Mr. Jai, Ms. Geetanjali Sehgal, Dr. O. S. Panwar and Mr. K. N. Sood for their help. ND acknowledges CSIR, Govt. of India and RKT acknowledge MNRE Govt. of India for providing financial support through SRF and JRF fellowships, respectively. 
Dwivedi et al. ACS Appl. Mater. Interfaces 5, 2725 (2013)

\section{REFERENCES}

(1) Talapatra, S.; Kar, S.; Pal, S. K.; Vajtai, R.; Cl, L.; Victor, P.; Shaijumon, M. M.; Kaur, S.; Nalamasu, O; Ajayan, P. M. Nature Nanotech. 2006, 1, 112-116.

(2) Connolly, T.; Smith, R. C.; Hernandez, Y.; Gun'ko, Y.; Coleman, J. N.; Carey, J. D. Small 2009, 5, 826-831.

(3) Varshney, D.; Rao, C. V.; Guinel, M. J. F.; Ishikawa, Y.; Weiner, B. R.; Morell, G. J. Appl. Phys. 2011, 110, 044324.

(4) Carey, J. D. Phil. Trans. R. Soc. Lond. A 2003, 361, 2891-2907.

(5) Dwivedi, N.; Kumar, S.; Malik, H. K. J. Appl. Phys. 2012, 112, 023518.

(6) Erdemir, A.; Donnet, C. J. Phys. D Appl. Phys. 2006, 39, 311.

(7) Dwivedi, N.; Kumar, S.; Tripathi, R. K.; Carey, J. D.; Malik, H. K.; Dalai, M. K. ACS Appl. Mater. Interfaces 2012, 4, 5309-5316.

(8) Dwivedi, N.; Kumar, S.; Rauthan, C. M. S.; Panwar, O. S. Plasma Process. Polym. 2011, $8,100-107$.

(9) Dwivedi, N.; Kumar, S.; Malik, H. K. ACS Appl. Mater. Interfaces 2011, 3, 4268-4278.

(10) Tordjman, M.; Bolker, A.; Saguy, C.; Baskin, E.; Bruno, P.; Gruen, D. M.; Kalish, R. Adv. Funct. Mater. 2012, 22, 1827-1834.

(11) May, P. W.; Hohn, S.; Ashfold, M. N. R.; Wang, W. N.; Fox, N. A.; Davis, T. J.; Steeds, J. W. J. Appl. Phys. 1998, 84, 1618-1625. 
Dwivedi et al. ACS Appl. Mater. Interfaces 5, 2725 (2013)

(12) Silva, S. R. P.; Carey, J. D. Diam. Relat. Mater. 2003, 12, 151-158.

(13) Varshney, D.; Makarov, V. I.; Saxena, P.; Berrios, A. G.; Scott, J. F.; Weiner, B. R.; Morell, G. Nanotech. 2010, 21, 285301.

(14) Dwivedi, N.; Kumar, S.; Carey, J. D.; Malik, H. K.; Govind. J. Appl. Phys. 2012, 112, 113706.

(15) llie, A.; Ferrari, A. C. ; Yagi, T.; Rodil, S. E. ; Robertson, J.; Barborini, E.; Milani, P. J. Appl. Phys. 2001, 90, 2024-2032.

(16) Silva, S. R. P.; Amaratunga, G. A. J.; Barnes, J. R. Appl. Phys. Lett. 1997, 71, 1477-1479.

(17) Forrest, R. D.; Burden, A. P.; Silva, S. R. P.; Cheah, L. K.; Shi, X. Appl. Phys. Lett. 1998, $25,3784-3786$.

(18) Carey, J. D.; Forrest, R. D.; Silva, S. R. P. Appl. Phys. Lett. 2001, 78, 2339-2341.

(19) Collins, M; Barklie, R.C.; Anguita, J.V.; Carey, J.D.; Silva S.R.P. Diam. Relat. Mater. 2000, 9, 781-785.

(20) Silva, S. R. P.; Carey, J. D.; Guo, X.; Tsang, W. M.; Poa, C. H. P. Thin Solid Films 2005, $482,79-85$.

(21) Carey, J. D.; Smith, R. C.; Silva, S. R. P. J. Mater. Sci.:Mater. Electron. 2006, 17, 405412.

(22) Carey, J. D.; Silva, S. R. P. Appl. Phys. Lett. 2001, 78, 347-349.

(23) Khan, R. U. A.; Carey, J. D.; Silva, S. R. P. Phys. Rev. B 2001, 63, 121201. 
Dwivedi et al. ACS Appl. Mater. Interfaces 5, 2725 (2013)

(24) Ahmed, S. F.; Moon, M. W.; Lee, K. W. R. Appl. Phys. Lett. 2008, 92, 193502.

(25) Deguchi, M.; Taomoto, A. Vacuum 2010, 84, 438-443.

(26) Pauleau, Y.; Theiry, F.; Barna, P. B.; Misjak, F.; Kovacs, A.; Dub, S. N.; Uglov, V. V.; Kuleshov, A. K. Rev. Adv. Mater. Sci. 2004, 6, 140-149.

(27) Dwivedi, N.; Kumar, S.; Malik, H. K. J. Appl. Phys. 2012, 111, 014908.

(28) Dwivedi, N.; Kumar, S.; Tripathi, R. K.; Malik, H. K.; Panwar, O. S. Appl. Phys. A 2011, $105,417-425$.

(29) Tamor, M. A.; Vassell, W. C. J. Appl. Phys. 1994, 76, 3823.

(30) Weiler, M.; Sattel, S.; Giessen, T.; Jung, K; Ehrhardt, H.; Veerasamy, V. S.; Robertson, J. Phys. Rev. B 1996, 53, 1594.

(31) Ferrari, A. C.; Robertson, J. Philos. Trans. R. Soc. Lond. A 2004, 362, 2477-2512.

(32) Ferrari, A. C.; Rodil, S. E.; Robertson, J. Phys. Rev. B 2003, 67, 155306.

(33) Rodil, S. E.; Ferrari, A. C.; Robertson, J.; Milne, W. I. J. Appl. Phys. 2001, 89, 5425-5430.

(34) Nilsson, L.; Groening, O.; Emmenegger, C.; Kuettel, O.; Schaller, E.; Schlapbach, L.; Kind, H.; Bonard, J.-M.; Kern, K. Appl. Phys. Lett. 2000, 76, 2071-2073. 
Dwivedi et al. ACS Appl. Mater. Interfaces 5, 2725 (2013)

\section{Figure Captions}

Figure 1: (a)-(d) Schematic representation of deposition of Ag dots containing DLC films.

Figure 2: SEM images of samples (a) ND-1, (b) ND-2, (c) ND-3, (d) ND-3 with a view of the dots in the corner, (e) ND-3 from a peak region, (f) ND-4 with view of the dots in corner, (g) ND-4 from a peak region and (h) sample ND-6.

Figure 3: AFM images of samples (a) ND-1, (b) ND-3 from the peak region, (c) ND-3 from the valley region, (d) ND-3 after field emission and (e) ND-6.

Figure 4: ToF-SIMS depth profiles of samples (a) ND-1, (b) ND-2, (c) ND-3 from the valley region, (d) ND-3 from the peak region, (e) ND-5 and (f) ND-6.

Figure 5: Visible Raman spectra of samples (a) ND-1, (b) ND-2, (c) ND-4, (d), ND-5 and (e) ND-6.

Figure 6: Variations of (a) $G$ peak position and (b) $I_{D} / I_{G}$ ratio for various ND samples.

Figure 7: (a) Field emission I-E characteristics and (b) their fitting to the Fowler - Nordheim model for various ND samples. 


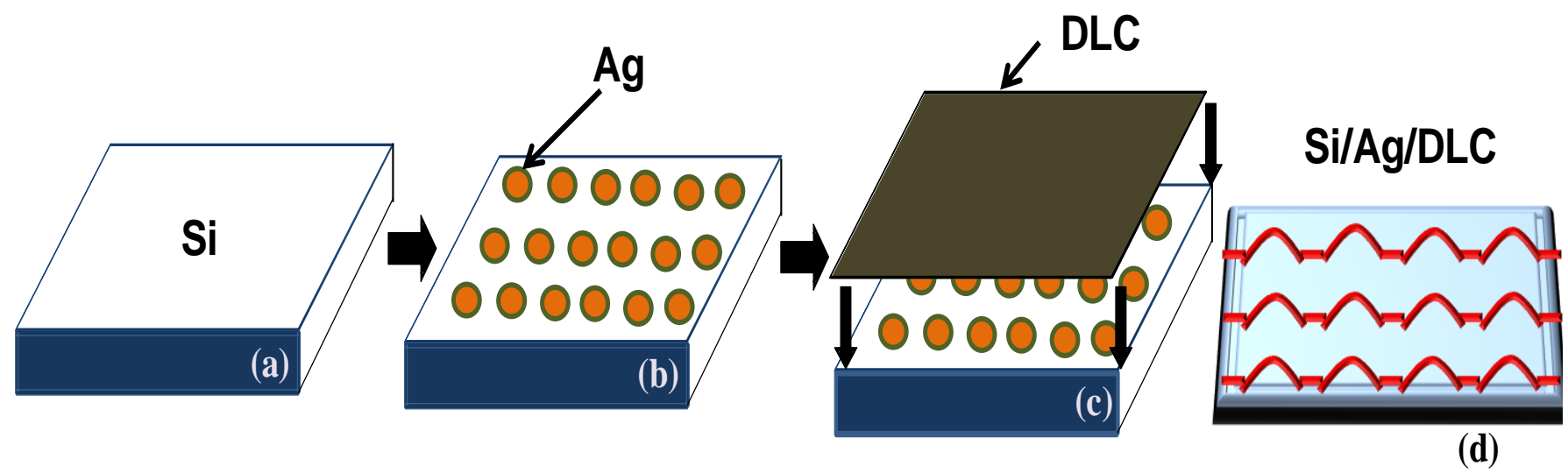

Fig. 1 

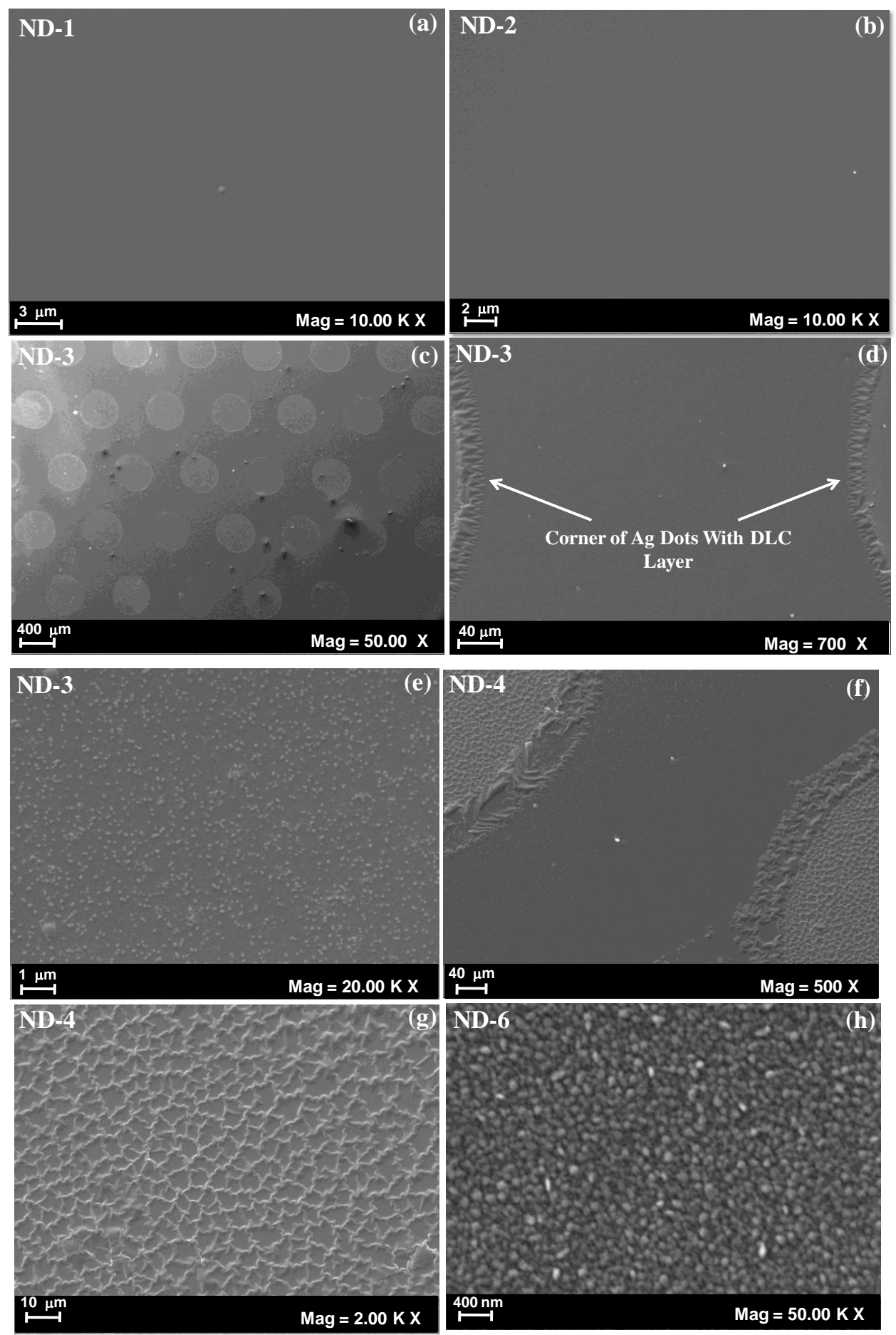

Fig. 2 

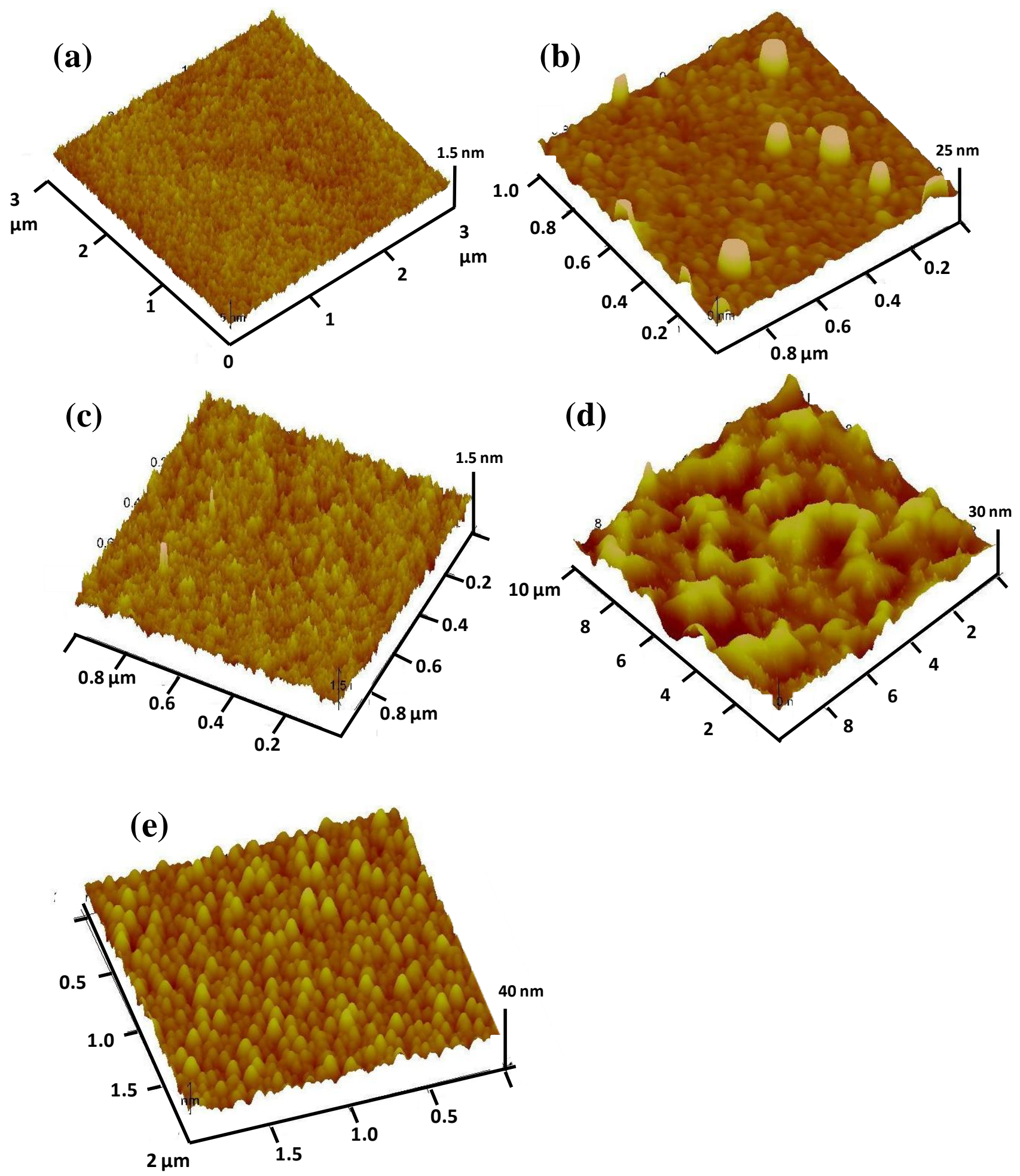

Fig. 3 

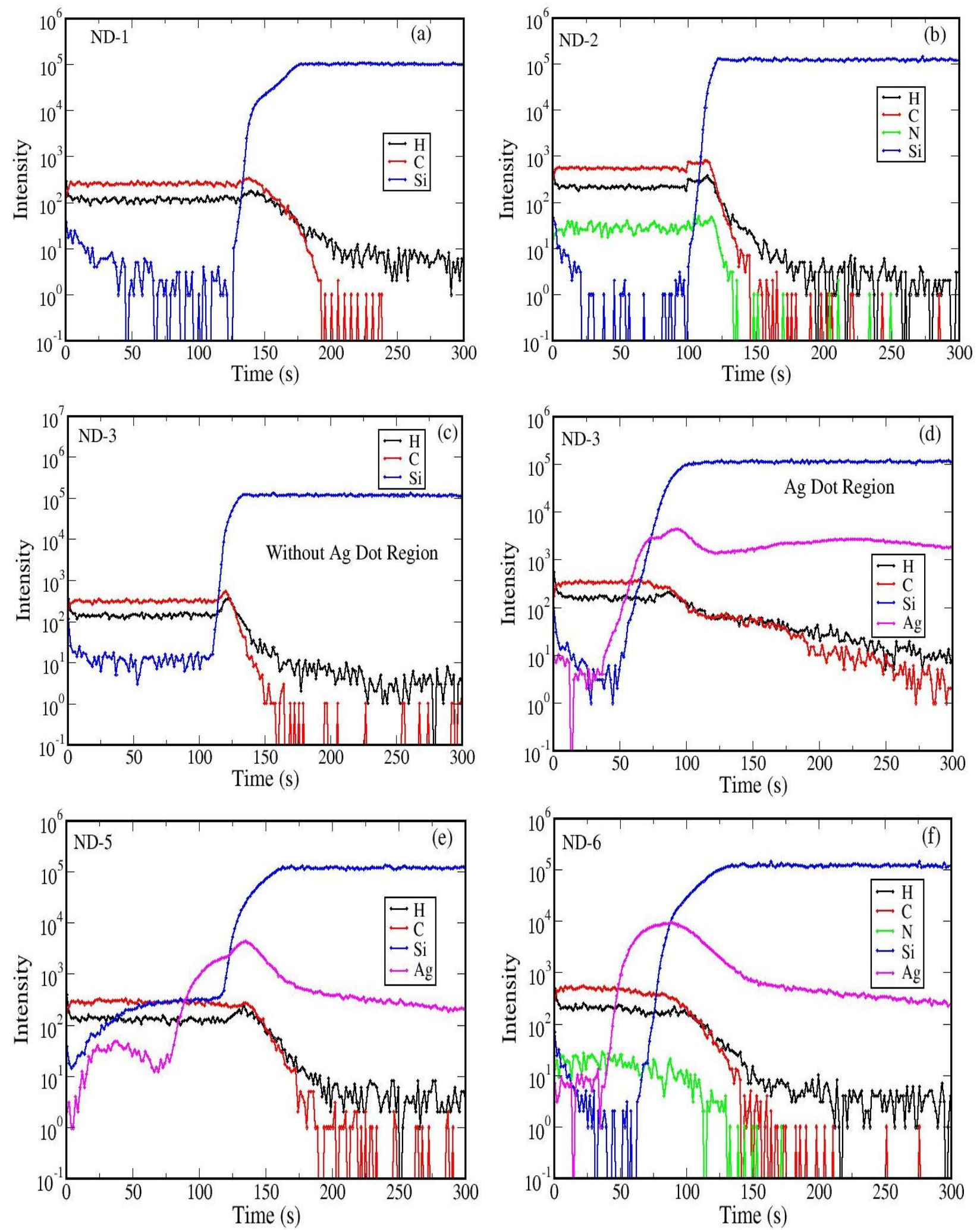

Fig. 4 


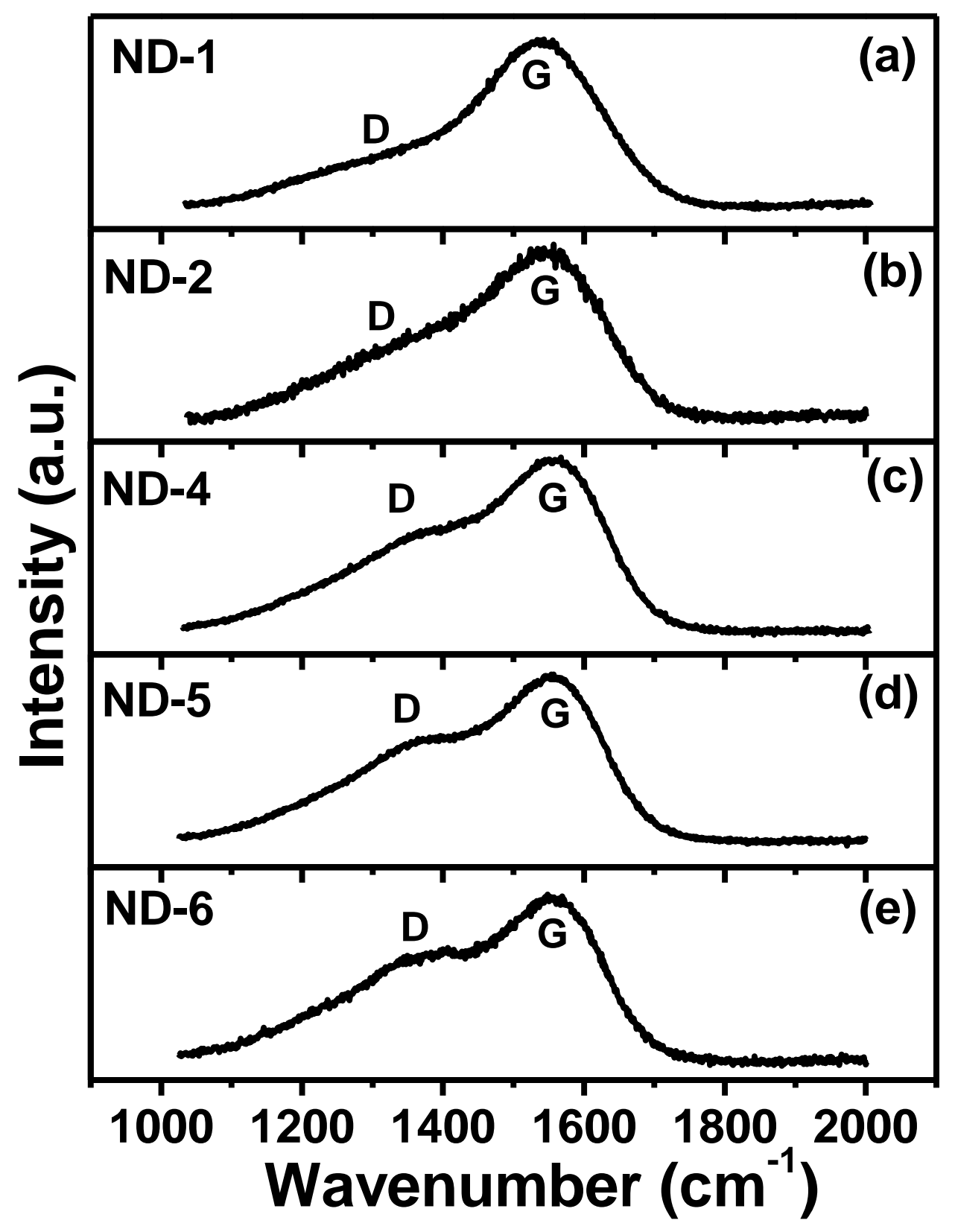

Fig. 5 

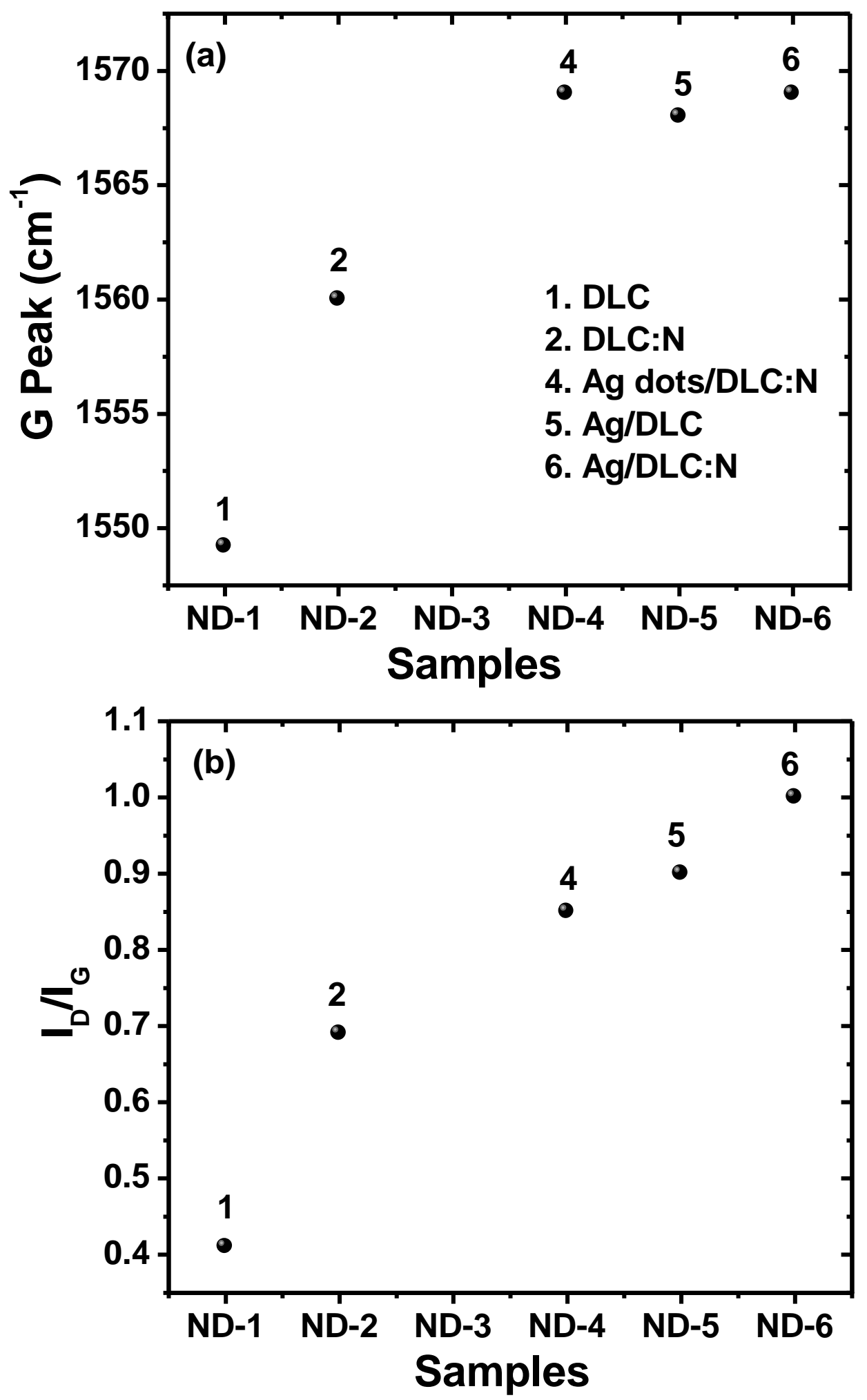

Fig. 6 

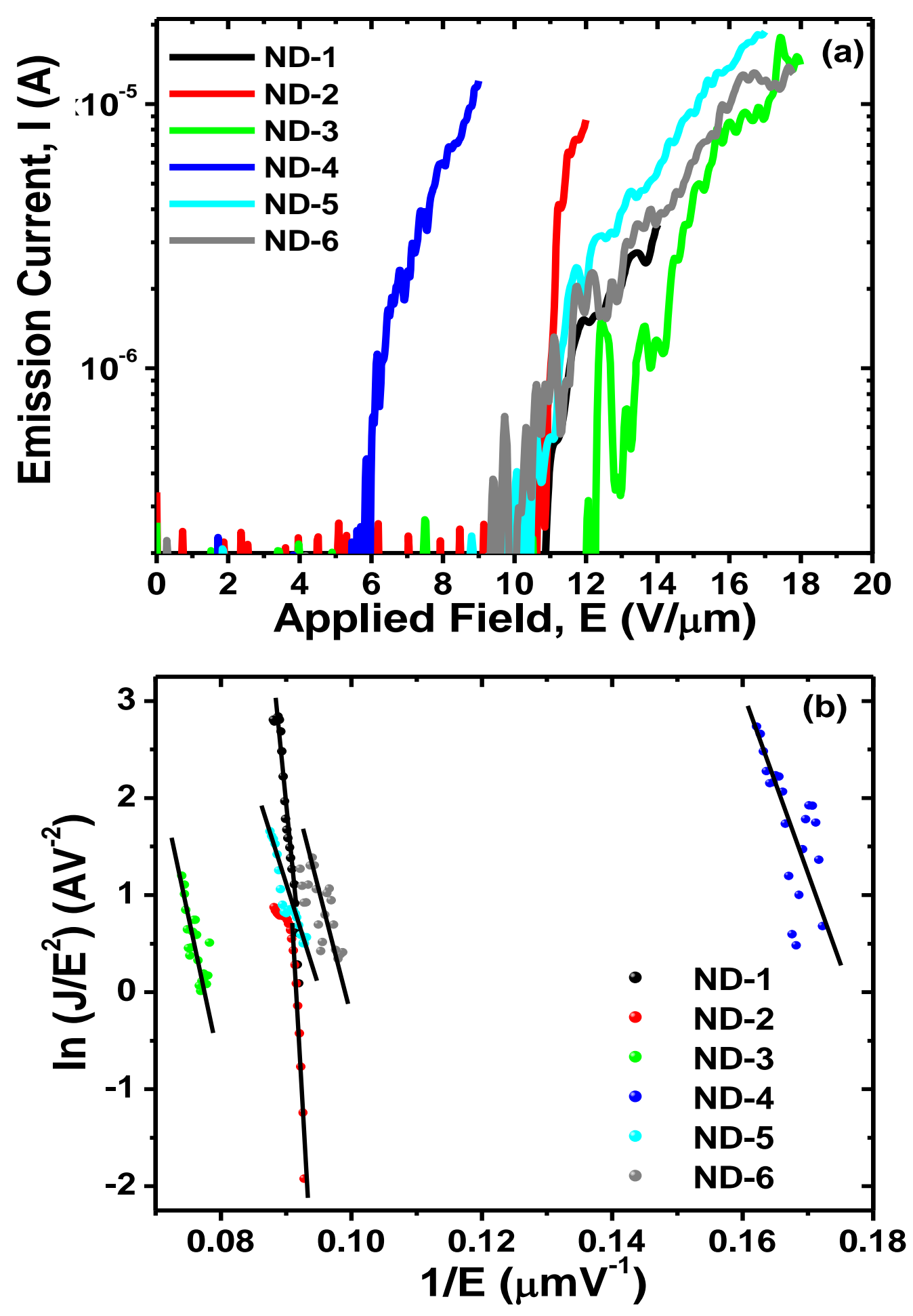

Fig. 7 


\section{Graphics}

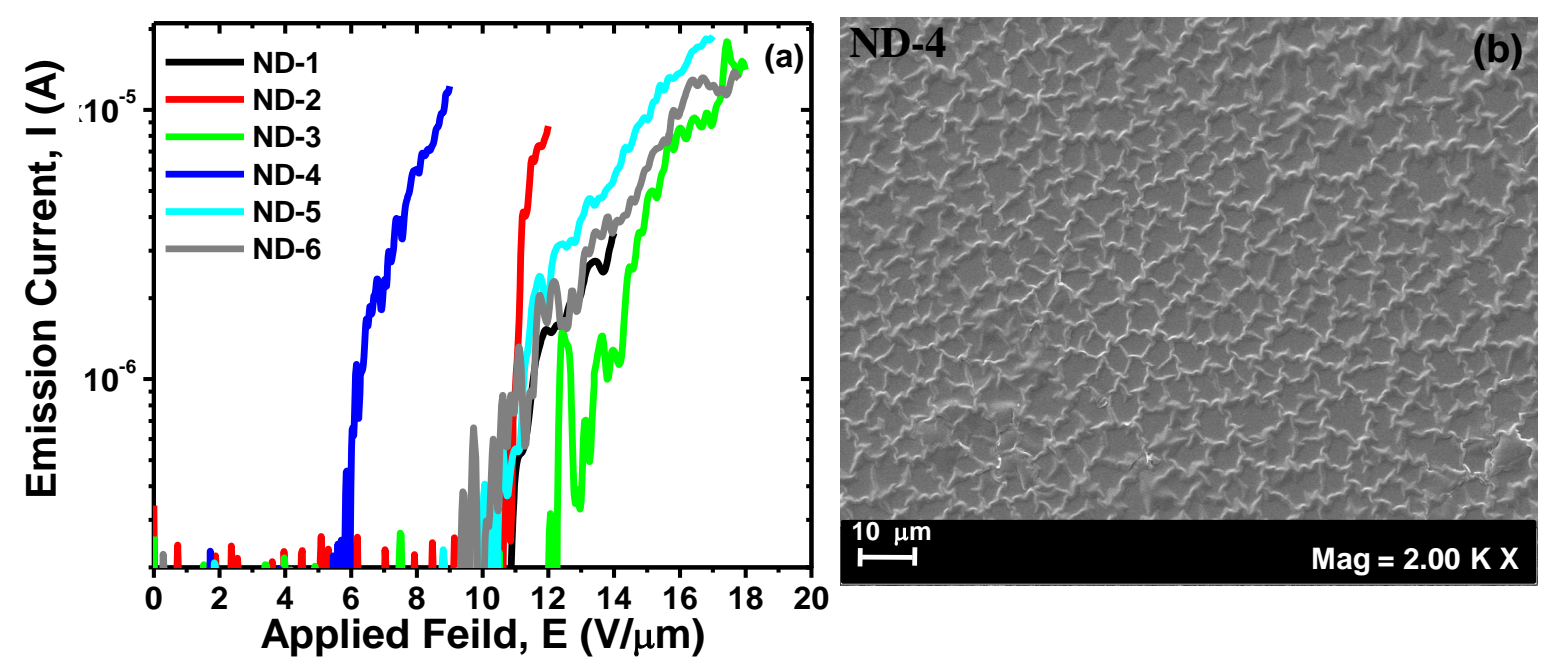

